\title{
Sexagem fetal em ovelhas Santa Inês por ultra-sonografia
}

\author{
Fetal sexing in Santa Inês ewes by ultrasonography
}

\section{Maico Henrique Barbosa dos Santos ${ }^{1}$ Érica Paes Barreto Xavier de Moraes ${ }^{1}$ Sebastião Inocêncio Guido ${ }^{1}$ Filipe Queiros Gondim Bezerra ${ }^{1}$ Arthur Nascimento Melo ${ }^{2}$ Paulo Fernandes de Lima $^{3}$ Marcos Antonio Lemos de Oliveira ${ }^{3}$}

\section{RESUMO}

O presente estudo teve a finalidade de identificar o sexo e de determinar o dia da migração do tubérculo genital (TG) de fetos ovinos através da ultra-sonografia em tempo real. O sexo foi identificado no Experimento I (EI) levando-se em consideração a localização do TG e no Experimento II (EII), a presença do pênis, prepúcio e bolsa escrotal no feto macho e das tetas, vulva e clitóris no feto fêmea. No EI, as fêmeas $(n=17)$ foram monitoradas em intervalos de 12 horas, do $35^{\circ}$ ao $46^{\circ}$ dia de gestação, por via transretal com transdutor linear $(6,0$ e 8,0 MHz). No EII, as fêmeas $(n=30)$ com gestação de 55 a 75 dias foram examinadas apenas uma vez, utilizandose o mesmo transdutor e via de exame do EI. Das 17 fêmeas do EI, $11(64,6 \%)$ tiveram seus fetos corretamente sexados, independente da gestação ter sido simples (7/11), dupla (3/11) ou tríplice (1/11). Nas $6(35,4 \%)$ gestações restantes, $3(17,7 \%)$ foram duplas, sendo impossível sexar um feto de cada gestação. Nas outras $3(17,7 \%)$ gestações, os fetos foram corretamente sexados, apesar dos nascimentos não coincidirem com a quantificação. Num feto macho de uma gestação simples, a migração ocorreu no $37^{\circ}$ dia e até o $46^{\circ}$, todos os fetos das outras gestações estavam corretamente sexados. Das 30 fêmeas do EII, 16 (53,4\%) apresentaram gestações simples e a acurácia da sexagem foi de $100 \%$. Nas 14 (46,6\%) restantes, as gestações foram duplas, sendo impossível, em quatro casos, determinar o sexo de, pelo menos, um dos gêmeos. De todos os fetos nascidos, a acurácia geral da sexagem foi de 88,0\% (EI) e 90,9\% (EII), não sendo observada diferença $(P>0,05)$ entre ambos os experimentos. Os resultados permitem concluir que a ultra-sonografia em tempo real é um método eficiente para diagnosticar o sexo fetal pela visualização do TG, assim como pela identificação do pênis, prepúcio e bolsa escrotal no feto macho e das tetas, vulva e clitóris no feto fêmea, desde que os exames sejam realizados a partir do 50음 dia de gestação. Palavras-chave: tubérculo genital, saco escrotal, pênis,
prepúcio, tetas, vulva.

\begin{abstract}
The present study aimed to identify the sex and to determine the day of genital tubercle (GT) migration of ovine fetuses using real time ultrasonography. The sex was identified in Experiment (EI) taking into consideration the localization of GT and in Experiment II (EII) the presence of penis, prepuce and scrotal bag in male fetus and nipples, genital swelling and clitoris in female fetus. In EI, the females $(n=17)$ were monitored with 12 hour intervals from the $35^{\text {th }}$ to the $46^{\text {th }}$ day of pregnancy, by transrectal via with linear transducer $(6.0$ and $8.0 \mathrm{MHz})$. In EII, the females $(n=30)$ with pregnancy period from 55 to 75 days were examined once only, using the same transducer and via used in EI. Among 17 females in EI, 11 (64.6\%) fetuses were correctly sexed independent of single (7/11), twin (3/11) or triple (1/11) pregnancy In 6 (35.4\%) pregnancies, 3 (17.7\%) were twins, being impossible to sex one fetus of each pregnancy. In other $3(17.7 \%)$ pregnancies the fetuses were correctly sexed, although the birth did not coincide with the quantification. In a male fetus of a single pregnancy, the migration of the GT began on day 37 of pregnancy and on the $46^{\text {th }}$ day all the fetuses of the other pregnancies were correctly sexed. Among 30 females in EII, 16 (53.4\%) pregnancies were single being sexed with accuracy of $100 \%$. In other 14 (46.6\%) remainder the pregnancies were twins, being impossible, in four cases, to be determined the sex of one of each twin. The incorrect diagnoses were fetuses sexed as females, however born as males. From the all born fetuses the total accuracy was $88.0 \%$ (EI) and $90.9 \%$ (EII), being not observed difference $(P>0.05)$ between both experiments. The results allow to conclude that
\end{abstract}

${ }^{1}$ Programa de Pós-graduação em Ciência Veterinária, Departamento de Medicina Veterinária, Universidade Federal Rural de Pernambuco (UFRPE). Av. Dom Manoel de Medeiros, s/n, 52171-900, Dois Irmãos, Recife, PE, Brasil.

${ }^{2}$ Curso de Medicina Veterinária, UFRPE, Recife, Pernambuco, Brasil

32Departamento de Medicina Veterinária, UFRPE, Recife, Pernambuco, Brasil. E - mail: maloufrpe@uol.com.br, malo@ufrpe.br. Autor para correspondência. 
ultrasonography in real time is an efficient method to diagnose the fetal sex by visualization of GT, as well as by identification of penis, prepuce and scrotal bag in male fetus and nipples, genital swelling and clitoris in female fetus, since the scanning are performed from Day 50 of pregnancy

Key words: genital tubercle, scrotal bag, penis, prepuce, nipples, genital swelling.

\section{INTRODUÇÃO}

A cadeia de eventos do processo produtivo tem na reprodução animal seu principal alicerce e qualquer alteração na esfera reprodutiva compromete o potencial produtivo do rebanho e interfere no custo/ benefício da atividade pecuária (BICUDO, 2002). Assim, para racionalizar a exploração da caprino-ovinocultura, faz-se necessária a adoção de ferramentas capazes de viabilizar o monitoramento reprodutivo para aumentar a produtividade do rebanho (SANTOS et al., 2004).

Nos últimos anos, foi gerada uma série de biotécnicas, como a inseminação artificial (IA), a transferência de embriões (TE) e até a fecundação in vitro (FIV), na expectativa de tecnificar a produção das criações mais estruturadas. Apesar dessas ferramentas contribuírem para aumentar a fecundidade, a prolificidade e acelerar o melhoramento genético dos rebanhos, é preciso considerar que a utilização destas biotécnicas requer alto investimento financeiro e necessita de um comércio atraente que assegure rotatividade e retorno do capital investido, pelo menos em médio prazo (BANDEIRA et al., 2004).

A identificação precoce do sexo fetal através da ultra-sonografia vem sendo implementada com sucesso e em larga escala, há alguns anos, em bovinos e eqüinos. Nos pequenos ruminantes, apesar de ser ainda de uso restrito (ANDRADE et al., 2004), pode contribuir para a evolução de diversas áreas da pesquisa fundamental, visando, por exemplo, o diagnóstico precoce do sexo de fetos gerados através da IA com sêmen sexado (JOHNSON, 2000; GARNER, 2001), por meio da TE com sexo pré-determinado (GUTIERREZADAN et al., 1997) ou através da FIV utilizando a técnica da injeção intracitoplasmática de espermatozóide (CATT et al., 1996).

Dentre as vantagens da sexagem fetal, podese destacar sua influência na produção animal, particularmente em rebanhos leiteiros, por permitir um melhor planejamento para adquirir e comercializar animais do próprio rebanho (HAIBEL, 1990). O resultado pode elevar ou diminuir o valor do feto sexado e facilitar a coordenação de ações que visem racionalizar produção e lucratividade, enfatizando que aprimorar o planejamento implica uma maior concentração de fêmeas nos rebanhos leiteiros e de machos nos de carne (REICHENBACH et al., 2004).

Apesar do exposto, a difusão da técnica de sexagem na cabra e na ovelha, sobretudo em condições de campo, depende de uma maior precisão no diagnóstico, uma vez que gestações múltiplas, disparidade na idade dos conceptos e, principalmente, a realização de um único exame, podem contribuir para a emissão de diagnósticos equivocados (BÜRSTEL, 2002).

Considerando que a sexagem fetal nos pequenos ruminantes ainda é restrita em todos os continentes, especialmente no Brasil e na Região Nordeste que detém a maior concentração dessas espécies, este trabalho objetivou identificar precocemente o sexo e determinar o período de migração do tubérculo genital (TG) de fetos da espécie ovina através da ultra-sonografia em tempo real.

\section{MATERIAL E MÉTODOS}

Neste trabalho, constituído de dois experimentos (EI e EII), foram utilizadas 47 ovelhas da raça Santa Inês pertencentes à Fazenda Pocinhos D’água, localizada no Município de Brejo da Madre de Deus, Região Agreste do Estado de Pernambuco.

Foi utilizado um aparelho ultra-sonográfico, modelo 240 Parus (Pie Medical), equipado com um transdutor linear (6,0 e 8,0 MHz) adaptado a um suporte de PVC para facilitar a manipulação no reto do animal, como sugerido por OLIVEIRA et al. (2004), além de uma impressora (Seikosha VP/1200 - Sony). Os exames ultra-sonográficos foram realizados por um único operador.

No EI $(n=17)$, as fêmeas foram monitoradas em intervalos de 12 horas, do $35^{\circ}$ ao $46^{\circ}$ dia de gestação, para acompanhar a migração do TG. Foram considerados fetos machos aqueles que apresentavam o TG em posição imediatamente caudal ao cordão umbilical (Figura 1A) e fêmeas, os que evidenciavam o TG posicionado abaixo da cauda (Figura 1B).

No EII, as 30 fêmeas com idade gestacional entre o $55^{\circ}$ e o $75^{\circ}$ dia foram submetidas a um exame único com o transdutor linear por via transretal. O sexo foi identificado tomando-se por base, a presença do TG ou de uma ou mais estruturas da genitália externa, como bolsa escrotal, pênis e prepúcio no feto macho (Figura 1C) e tetas, vulva e clitóris (Figura 1D) no feto fêmea.

Os dados foram analisados através do teste de Qui-quadrado, considerando-se o nível de significância da ordem de 5\%. 


\section{RESULTADOS E DISCUSSÃO}

No EI, o acompanhamento diário permitiu registrar, em um animal, o início da migração do TG em direção à região umbilical a partir do 37º dia de gestação, mas somente no $46^{\circ}$ dia é que a identificação do sexo de todos os animais foi concluída (Figura 2). O valor médio do dia da migração foi de 41,4 $\pm 2,1$ dias. Das 17 fêmeas, $11(64,6 \%)$ tiveram seus fetos sexados corretamente, independente da gestação ter sido simples (7/11), dupla (3/11) ou tríplice (1/11). Nas 6 (35,4\%) gestações restantes, 3 (17,7\%) foram duplas e não foi possível sexar um feto de cada gestante. Nas outras $3(17,7 \%)$, os fetos foram corretamente sexados, apesar dos nascimentos não coincidirem com a quantificação.

Das 30 fêmeas do EII, 16 (53,4\%) apresentaram gestações simples e nestas, a acurácia da sexagem foi de $100 \%$. Nas $14(46,6 \%)$ restantes que evidenciaram gestação dupla, em quatro, foi impossível determinar o sexo de um dos gêmeos (Tabela 1). De todos os fetos nascidos, a acurácia total foi de $88,0 \%$ (EI) e 90,9\% (EII), não sendo observada diferença $(\mathrm{P}>0,05)$ entre ambos os experimentos.
É comum existir a necessidade da realização de exames ultra-sonográficos seriados, seja em intervalos de 24, 48 ou 72 horas ou mesmo até em intervalos mais longos, para diagnosticar o sexo do feto com precisão, como sugerem BÜRSTEL (2002) e REICHENBACH et al. (2004). Neste trabalho, contudo, foi demonstrado que exames repetidos em pequenos intervalos, não proporcionam resultados mais expressivos, apesar de permitir, no feto macho, a visualização precoce do TG em migração ou já devidamente posicionado na região umbilical. Além disso, é preciso considerar que exames realizados em curtos intervalos, sejam de 24 ou de 48 horas e especialmente como o adotado neste trabalho, certamente são inviáveis nas condições de campo. Os constantes deslocamentos e a distância que o operador deve percorrer com as freqüentes visitas técnicas oneram o custo/benéficio dos exames ultrasonográficos, inviabilizando uma atividade que, se bem administrada, é de grande importância para a produção animal em decorrência de maximizar as práticas de manejo e de planejamento comercial da propriedade, conforme reportaram HAIBEL (1990) e REICHENBACH et al. (2004).

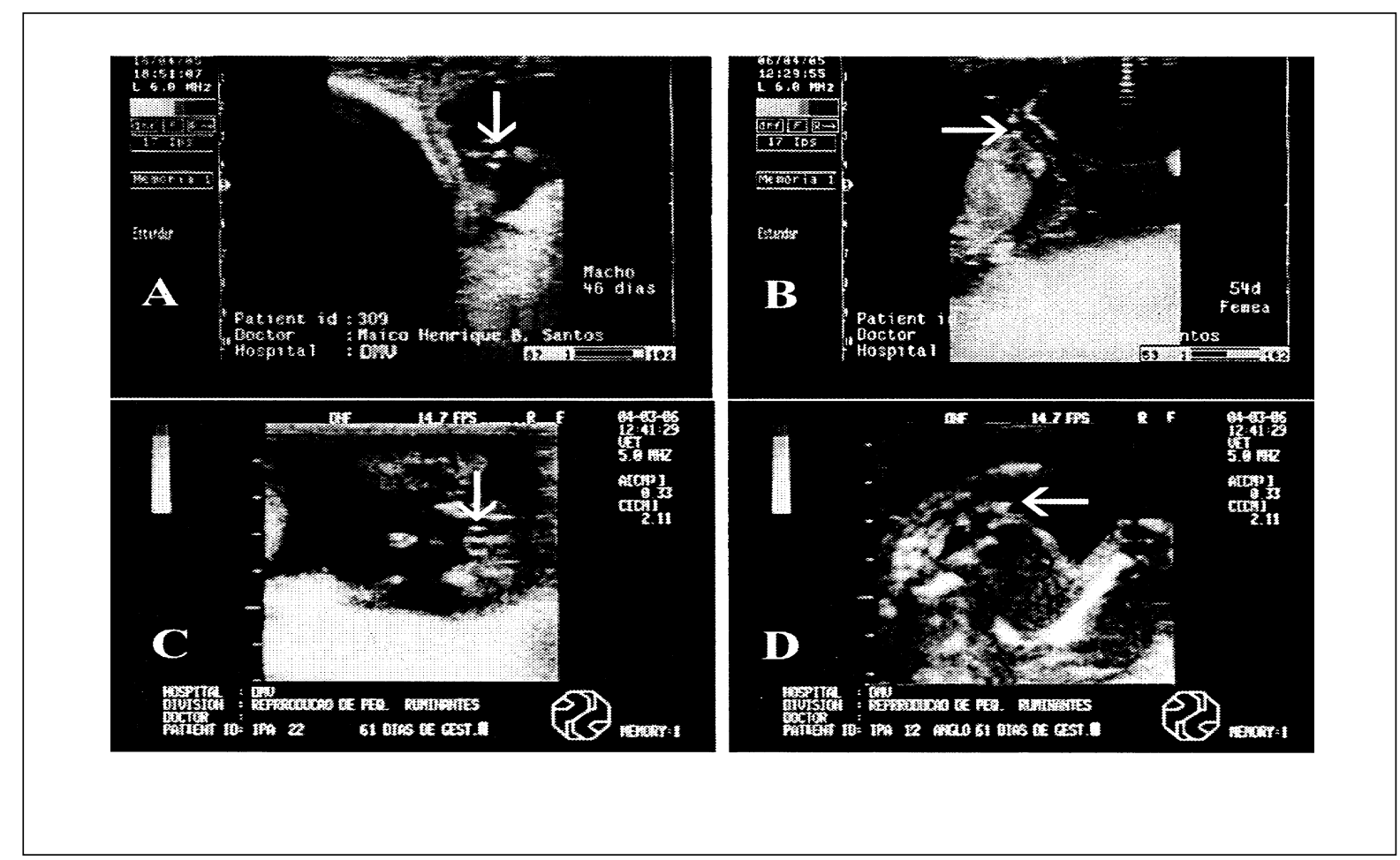

Figura 1 - Imagens do posicionamento do TG (i) no feto macho (A) e (g) na fêmea (B). Feto macho (C) mostrando a bolsa escrotal (i) e o prepúcio (h) e outro feto fêmea (D) evidenciando a vulva (f).

Ciência Rural, v.36, n.2, mar-abr, 2006. 


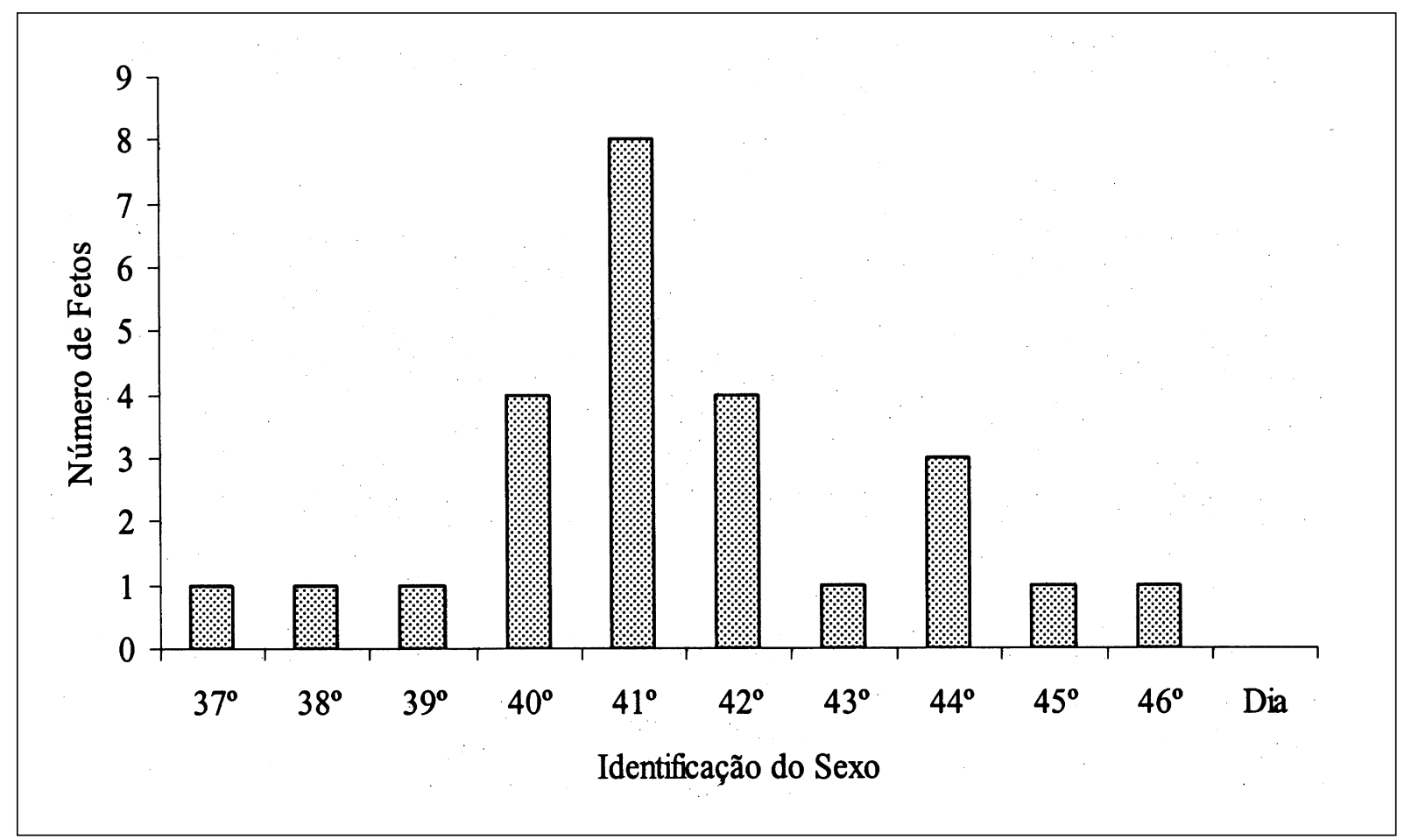

Figura 2 - Monitoramento diário da sexagem fetal levando em consideração o posicionamento do tubérculo genital (TG). O feto que apresentava o TG localizado caudalmente ao cordão umbilical era diagnosticado como macho e o que evidenciava o TG posicionado próximo à cauda era sexado como fêmea.

Além do abordado e na dependência do período e do tipo de gestação, nem sempre é possível quantificar todos os fetos de forma precisa, como por exemplo, nas gestações múltiplas e, muito menos, identificar o sexo de todos. Abordagem semelhante foi anteriormente descrita por REICHENBACH et al. (2004), ao explicitarem que, além da necessidade de equipamentos mais sofisticados e de pessoal qualificado, um outro fator que limita a identificação do sexo fetal em condições de campo é a necessidade de exames seriados, principalmente nos casos de gestações múltiplas.

A observação do TG em migração aos 37 dias de gestação ocorreu apenas em um feto macho, ressaltando que este mesmo feto apresentou, ao nascimento, peso e tamanho diferenciado dos demais. É permissível especular que o componente genético, associado a outros fatores, tenha contribuído para tal precocidade. Como exemplo, pode ser mencionado o fato de ter sido produto de gestação simples e, devido a isso, ter sido condição privilegiada para desenvolver. Os achados deste trabalho contrariam as observações de BÜRSTEL (2002) ao relatarem que somente após o $40^{\circ}$ dia de gestação é possível visualizar o TG através da ultra-sonografia. Admite-se que a controvérsia entre ambos os resultados deve ser decorrente do efeito da precocidade da raça ou como já ressaltado anteriormente, em conseqüência da qualidade do equipamento.

Mesmo não existindo diferença entre a sexagem de fetos provenientes de gestações simples ou duplas, as maiores dificuldades na sexagem ocorrem naquelas com mais de um feto, achados que reforçam as considerações de WHITE et al. (1984), GEARHART et al. (1988) e HAIBEL (1990), bem como o relato de BÜRSTEL et al. (2001) ao propor, nos casos de gestações múltiplas, a realização de exames em dois períodos consecutivos, sendo o primeiro realizado entre o $50^{\circ}$ e o $56^{\circ}$ dia e o segundo entre o $66^{\circ}$ e o $70^{\circ}$ dia. Advertiram ainda que exames para sexagem fetal somente devem ser implementados nos casos de gestação múltipla com até três fetos, em função dos diagnósticos resultantes desses exames serem vulneráveis a freqüentes equívocos, opinião compartilhada com WHITE et al. (1984), GEARHART et al. (1988) e HAIBEL (1990). Com relação às gestações duplas, nas quais a quantificação não coincidiu com o nascimento, é importante salientar que, nos três casos, um dos gêmeos sofreu um processo de mumificação, ocorrência que foi registrada ao serem examinados os envoltórios fetais imediatamente após o parto. Além disso, é interessante ressalvar que as imagens de todos 
Tabela 1 - Identificação do sexo de fetos em fêmeas com gestação de 55 a 75 dias, através de exame ulta-sonográfico único.

\begin{tabular}{|c|c|c|c|c|c|c|}
\hline & \multicolumn{2}{|c|}{ Fetos sexados } & \multicolumn{2}{|c|}{ Fetos não sexados } & \multirow[b]{2}{*}{ Fetos Nascidos } & \multirow[b]{2}{*}{$\begin{array}{c}\text { Acurácia do diagnóstico } \\
\mathrm{n}(\%)\end{array}$} \\
\hline Gestação & Macho n & Fêmea n & Macho $n$ & Fêmea n & & \\
\hline Simples & 10 & 6 & - & - & 16 & $16 / 16(100,0)$ \\
\hline Dupla & 10 & 14 & 2 & 2 & 28 & $24 / 28(85,7)$ \\
\hline Total & 20 & 20 & 2 & 2 & 44 & $40 / 44(90,9)$ \\
\hline
\end{tabular}

os diagnósticos eram impressas, razão pela qual é possível afirmar não ter havido equívoco na quantificação dos conceptos.

As porcentagens de acerto equivalentes a $100 \%$ obtidas nas gestações simples foram maiores do que os índices reportados por COUGHBROUGH \& CASTELL (1998) e BÜRSTEL (2002), salientando-se que os primeiros autores ainda relataram ter sido impossível determinar o sexo fetal em $7 \%$ dos animais examinados por via transretal. A apresentação do concepto no exato momento da avaliação e o número de exames efetuados num mesmo animal são fatores decisivos para visualização das estruturas anatômicas.

Os resultados acima apresentados permitem o comentário de que a via transretal é eficiente para a sexagem entre o $46^{\circ}$ e o $75^{\circ}$ dia de gestação, independentemente de ser simples ou dupla. Ainda é possível enfatizar que esse resultado contraria o de BÜRSTEL et al. (2002), que relataram não ser recomendado utilizar essa via de exame nas gestações múltiplas. A experiência do operador e a qualidade do equipamento ultra-sonográfico disponível, como já anteriormente ressaltadas, são fundamentais para o êxito do diagnóstico do sexo fetal em qualquer período de gestação. No caso específico deste trabalho, ao se utilizar um transdutor linear com dupla freqüência, é permissível admitir que o equipamento deve ter contribuído para os resultados terem sido mais expressivos do que os de COUGHBROUGH \& CASTELL (1998) e de BÜRSTEL (2002).

Determinar o dia exato em que ocorre a migração do TG ainda é o maior desafio da sexagem fetal nos pequenos ruminantes. Mesmo considerando que a sexagem fetal com base na localização do TG já

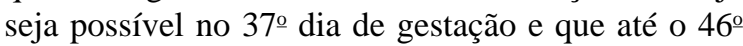
todos os fetos podem estar devidamente sexados na raça Santa Inês, sugere-se que o exame ultrasonográfico deva ser realizado somente a partir do $50^{\circ}$ dia após a cobertura. Essa proposição tem a finalidade de evitar que fetos machos sejam indevidamente sexados como fêmeas, porque existe a possibilidade da migração do TG ocorrer de forma mais tardia. Apesar do exposto, ainda é preciso enfatizar que o TG de algum feto pode migrar, mesmo que remotamente, após o $50^{\circ}$ dia, em conseqüência de possíveis variações entre indivíduos da mesma e de diferentes raças, assim como entre fetos originados de monta natural e de transferência de embriões congelados, ao menos em fetos da raça Morada Nova (SANTOS, 2005 - Informe verbal).

\section{CONCLUSÃO}

Finalmente, é permitido concluir que a ultrasonografia em tempo real é um método eficiente tanto para diagnosticar o sexo fetal pela visualização do TG, quanto pela identificação das estruturas relativas à genitália externa, desde que os exames sejam realizados a partir do $50^{\circ}$ dia de gestação.

\section{FONTES DE AQUISIÇÃO}

SANTOS, M.H.B. Programa de Pós-graduação em Ciência Veterinária da UFRPE. Av. Dom Manoel de Medeiros, s/n, Dois Irmãos, 52171-900, Recife-PE/Brasil (maico.henrique@uol.com.br). Angra dos Reis, XIX Reunião Anual da SBTE, 25 a 28 de agosto de 2005, Work Shop Aspectos práticos da IA e TE em Caprinos e Ovinos.

\section{REFERÊNCIAS}

ANDRADE, J.C.O et al. Sexagem fetal em ovinos. Acta Scientiae Veterinariae, v.32, p.185, 2004.

BANDEIRA, D.A. et al. Aspectos da caprino-ovinocultura no Brasil e seus reflexos produtivo e reprodutivo. In: SANTOS, M.H.B. et al. Diagnóstico de gestação na cabra e na ovelha. São Paulo: Varela, 2004. Cap.1. p.1-9.

BICUDO, S.D. Sumários de pesquisas. In: SIMPÓSIO PAULISTA DE OVINOCUlTuRA, 6., 2002, Botucatu. Anais... Botucatu: Associação Paulista dos Criadores de Ovinos, 2002. p.88-100

BÜRSTEL, D. Untersuchungen zur intrauterinen Geschlechtsfeststellung bei Feten kleiner Wiederkäuer mittels Ultrasonographie. 2002. 142f. Tese (Doutorado em Medicina Veterinária) - Institut für Reproduktionsmedizin, Tierärztliche Hochschule Hannover.

BÜRSTEL, D. et al. Ultrasonographic determination of fetal sex in small ruminants. In: ANNUAL CONFERENCE OF THE 
EUROPEAN. SOCIETY FOR DOMESTIC ANIMAL REPRODUCTION, 5., 2001, Vienna. Proceeding... Vienna: ESDAR Newsletter, 2001. v.6, p.53-54.

BÜRSTEL, D. et al. Ultrasonographic diagnosis of fetal sex in small ruminants bearing multiple fetuses. Veterinary Record, v.151, n.21, p.635-636, 2002

CATT, S.L. et al. Birth of a male lamb derived from an in vitro matured oocyte fertilised by intracytoplasmic injection of a single presumptive male sperm. Veterinary Record, v.139, p.494-495, 1996.

Coughbrough, C.A.; CASTELl, M.C. Fetal sex determination by ultrasonically locating the genital tubercle in ewes. Theriogenology, v.50, p.263-267, 1998.

GARNER, D.L. Sex-sorting mammalian sperm: concept to application in animals. Journal of Andrology, v.22, p.519526, 2001.

GEARHART, M.A. et al. Real-time ultrasonography for determining pregnancy status and viable fetal numbers in ewes. Theriogenology, v.30, 323-337, 1988.

GUTIERREZ-ADAN, A. et al. Ovine-specific Y-chromosome RAPD-SCAR marker for embryo sexing. Animal Genetic, v.28, p.135-138, 1997.
HAIBEL, G.K. Use of ultrasonography in reproductive management of sheep and goat herds. Veterinary Clinics of North America: Food Animal Practice, v.3, p.597-613, 1990.

JOHNSON, L.A. Sexing mammalian sperm for production of offspring: the state-of-the-art. Animal Reproduction Science, v.60-61, p.93-107, 2000.

OLIVEIRA, M.A.L. et al. Aplicabilidade do scan B na reprodução de pequenos ruminantes. In: SANTOS, M.H.B. et al. Diagnóstico de gestação na cabra e na ovelha. São Paulo: Varela, 2004. Cap.13. p.85-96.

REICHENBACH, H-D. et al. Sexagem fetal na cabra e na ovelha por ultra-sonografia. In: SANTOS, M.H.B. et al. Diagnóstico de gestação na cabra e na ovelha. São Paulo: Varela, 2004. Cap.15. p.117-136.

SANTOS, M.H.B. et al. Diagnóstico de gestação por ultrasonografia de tempo real. In: _____. Diagnóstico de gestação na cabra e na ovelha. São Paulo: Varela, 2004. Cap.14. p.97-116.

WHITE, I.R. et al. Real-time ultrasonic scanning in the diagnosis of pregnancy and determination of fetal numbers in sheep. Veterinary Record, v.115, p.140-143, 1984. 